\title{
TU/e emonownen

\section{Multivalued geodesic ray-tracing for computing brain connections using diffusion tensor imaging}

\section{Citation for published version (APA):}

Sepasian, N., Thije Boonkkamp, ten, J. H. M., Haar Romenij, ter, B. M., \& Vilanova, A. (2012). Multivalued geodesic ray-tracing for computing brain connections using diffusion tensor imaging. SIAM Journal on Imaging Sciences, 5(2), 483-504. https://doi.org/10.1137/110824395

DOI:

10.1137/110824395

Document status and date:

Published: 01/01/2012

\section{Document Version:}

Publisher's PDF, also known as Version of Record (includes final page, issue and volume numbers)

\section{Please check the document version of this publication:}

- A submitted manuscript is the version of the article upon submission and before peer-review. There can be important differences between the submitted version and the official published version of record. People interested in the research are advised to contact the author for the final version of the publication, or visit the $\mathrm{DOI}$ to the publisher's website.

- The final author version and the galley proof are versions of the publication after peer review.

- The final published version features the final layout of the paper including the volume, issue and page numbers.

Link to publication

\section{General rights}

Copyright and moral rights for the publications made accessible in the public portal are retained by the authors and/or other copyright owners and it is a condition of accessing publications that users recognise and abide by the legal requirements associated with these rights.

- Users may download and print one copy of any publication from the public portal for the purpose of private study or research.

- You may not further distribute the material or use it for any profit-making activity or commercial gain

- You may freely distribute the URL identifying the publication in the public portal.

If the publication is distributed under the terms of Article 25fa of the Dutch Copyright Act, indicated by the "Taverne" license above, please follow below link for the End User Agreement:

www.tue.nl/taverne

Take down policy

If you believe that this document breaches copyright please contact us at:

openaccess@tue.nl

providing details and we will investigate your claim. 


\title{
Multi-valued Geodesic Based Fiber Tracking for Diffusion Tensor Imaging
}

\author{
N. Sepasian ${ }^{1}$, J.H.M. ten Thije Boonkkamp ${ }^{2}$, \\ A. Vilanova ${ }^{1}$, and B. M. Ter Haar Romeny ${ }^{1}$ \\ 1 Department of Biomedical Technology, \\ Eindhoven University of Technology, The Netherlands, \\ 2 Department of Mathematics and Computer Science, \\ Eindhoven University of Technology, The Netherlands. \\ \{N.Sepasian, J.H.M.tenThijeBoonkkamp, A.Vilanova, B.M.terHaarRomeny\}@tue.nl
}

\begin{abstract}
In this paper, we propose a new geodesic based algorithm for diffusion tensor fiber tracking. This technique is based on computing multi-valued solutions from the Euler-Lagrange form of the geodesic equations. Compared to other geodesic based approaches, multi-valued solutions at each grid point have been considered other than just computing the viscosity solution. This allows us to compute fibers in a region with sharp orientation, or when the correct physical solution is not the fiber computed from the first arrival time. Compared to the classical stream-line approach, our approach is less sensitive to noise, since the complete tensor is used. We also compare our algorithm with the PDE approach, using the Hamilton-Jacobi equation. We show that in the cases where the U-shaped bundles appear, our algorithm can capture the underlying fiber structure while other approaches may fail. The results for a realistic synthetic data field is shown for both methods.
\end{abstract}

Keywords: Geodesic, Diffusion tensor images, Fiber tracking, Hamilton-Jacobi equation, Euler-Lagrange equation.

\section{Introduction}

Diffusion tensor imaging (DTI) is the first non-invasive technique for reconstructing the underlying white matter bundles inside the brain [1]. Numerous algorithms have been introduced for reconstructing fiber bundles from DTI. In the classic fiber tracking algorithm, the fiber bundles are generated by using the principal direction of the diffusion tensor. Here, the fiber tracking stops if there is a sharp turn in the trajectory or when the fiber enters a region of low anisotropy. However, due to acquisition noise these methods may fail to track fibers; see Jun et al. [2].

Another class of algorithms is based on the front propagation algorithm using full tensor information. In these techniques the geodesic is computed in a Riemannian manifold by defining the metric as the inverse of the diffusion tensor. 
The standard eikonal equation is solved by applying the connectivity measurement as a cost function. In early publications, the cost function is based on the diffusion tensor similarity measurements and the front is evolved with the proportional speed between the front normal and the tensor dominant eigenvector. However, this is prone to incorrect propagation in anisotropic domains; see Parker et al. [3]. Jackowski et al. [4] represent the anisotropic cost function for front propagation, which is suitable for oriented domains. The propagation speed of the front is given by the diffusivity rate in the normal direction of the front; the fibers are extracted by backtracking the characteristics of the front rather than the normal.

The recent trend is to use high angular resolution DTI rather than regular DTI. Melonakos et al. [5] introduce geodesic fiber tracking by including the diffusivity direction in the cost function definition. Since the Riemannian metric is restricted to ellipsoidal profiles, the Finsler metric is used. The cost function to be minimized satisfies the solution of the steady Hamilton-Jacobi (HJ) equation. In this paper, we develop an algorithm for correcting geodesic fiber tracking for the second order diffusion tensor, which is still most commonly used.

One characteristic of the HJ equation is that it gives only the single-valued viscosity solution corresponding to the minimizer of the cost function. It is also well-known that the solution of HJ equation can develop discontinuities in the gradient field. These discontinuities happen when the correct solution becomes multi-valued. Therefore, developing the algorithm that can tackle these issues becomes important. Parker et al. [6] shows that some structures (e.g., Broca and Wernicke) have multiple path connections. Moreover, Jbabdi et al. [7] show that the U-shaped fibers correspond to the short association tracts, which can not be captured by HJ-based algorithms.

In this paper, we present a new algorithm for geodesic based fiber tracking that can tackle problems appearing in fiber tracking using HJ-based methods. Our algorithm solves the Euler-Lagrange (EL) form of the geodesic equations, using well-known Ordinary Differential Equation (ODE) solvers. This allows us to capture multi-valued geodesics connecting two given points. The EL framework gives us more insight into the local structure of the field. Therefore, in grid points where discontinuities or multi-phase solutions appear, all solutions can be captured. Once all geodesics are computed, one can filter out weak connections, using existing connectivity measures; see Sepasian et al. [8] and Astola et al. [9].

\section{Related Work}

Let us start with the linear wave equation, i.e.,

$$
\varphi_{t t}(x, t)=c(x)^{2} \nabla^{2} \varphi(x, t)
$$

where $c(x)$ is the local wave speed. The solution of (1) can be approximated by a simple wave of the form,

$$
\varphi(x, t) \approx A(x, t) e^{i \omega u(x, t)},
$$


a)

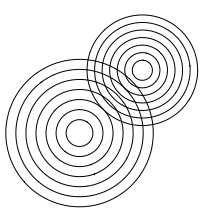

b)

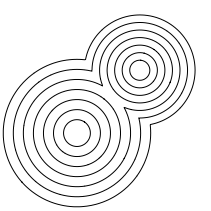

c)

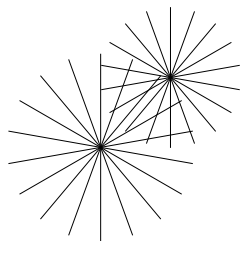

Fig. 1. The example of differences in computed solutions of wave propagation using different methods. a) Correct solution, b) Eikonal equation, c) Ray tracing

where, $A$ is an amplitude, $\omega$ is a frequency, and $u(x, t)$ is a phase function. Level curves of $\varphi$ correspond to wave fronts. Locally, the generic solution of (1) is the sum of solutions like (2). Generally speaking, this setting only breaks down at a few points, such as focus points, caustics and discontinuities in the gradient field. For the level set equation, this discontinuity appears where the correct solution becomes multi-valued, see figure 1 . Therefore, in such points the viscosity solution does not always agree with the correct physical solution and it always picks out the solution corresponding to the first arrival time.

The phase function $u(x, t)$ in (2) satisfies the nonlinear HJ type equation,

$$
u_{t}+c(x)|\nabla u|=0,
$$

where $|$.$| denotes the Euclidean norm in \mathbb{R}^{d}$. This equation may develop the multi-valued solution when two fronts collide. Those regions are called shocks. Therefore, the viscosity solution is needed to ensure the existence and uniqueness of the solution.

In DTI fiber tracking, the propagation of the front in an anisotropic field is considered. Different methods have been derived based on the level set equation. One of the popular methods is using the diffusion tensor as the inverse of the Riemannian metric. For the sake of completeness, we start with the definition of the metric in Riemannian geometry. For a $d$-dimensional manifold $\mathcal{M}$, a Riemannian metric is the collection of inner products $\left.<_{.,}.\right\rangle_{p}$ defined for every point $p$ on $\mathcal{M}$. These inner products denote the tangent space $\mathcal{T}_{p} \mathcal{M}$ of $\mathcal{M}$ at $p$ and provide the natural way to measure the length of tangent vectors to $\mathcal{M}$ at each location $p$. The tangent space $\mathcal{T}_{p} \mathcal{M}$ is a $d$-dimensional real vector space, attached to $p$, which contains all the possible directions of curves passing through $p$. Any element of the tangent space is expressed as $\sum \boldsymbol{x}^{i} \frac{\partial}{\partial \boldsymbol{x}^{i}}$, where $\frac{\partial}{\partial \boldsymbol{x}^{i}}$ are the basis vectors. Then the inner products $<\frac{\partial}{\partial \boldsymbol{x}^{i}}, \frac{\partial}{\partial \boldsymbol{x}^{j}}>$ yield a $d \times d$ symmetric, bilinear, positive definite form $G=\left(g_{i j}\right)$, known as the Riemannian metric. Therefore, the inner product of two tangent vectors $u$ and $v$ in $\mathcal{T}_{p} \mathcal{M}$ is expressed as

$$
<u, v>_{p}=u^{T} G v .
$$

Once the metric is defined, the notion of geodesic on a Riemannian manifold $\mathcal{M}$ can be determined, as defined by Lenglet et al. [10].

A geodesic between two points is the smooth curve whose length is the minimum of all possible lengths. In terms of variational calculus, the geodesic $\underline{x}(\tau)$ 
connecting two point $\underline{x}_{0}=\underline{x}(0)$ and $\underline{x}_{J}=\underline{x}(J)$ is the curve minimizing the functional,

$$
L(\underline{x})=\int_{0}^{\mathcal{T}} \sqrt{\underline{\dot{x}} G \underline{\dot{x}}} d \tau .
$$

The functional (4) satisfies both the HJ and the EL equation. They both give the geodesic equation as result, although using the HJ equation gives more global insight into the structure of the manifold. Concerning the local coordinates on $\mathcal{M}$, the EL geodesic equation is derived. For details we refer to Runborg [11].

There are several choices possible for the Hamiltonian. Jackowski et al. [4] introduced the Hamiltonian

$$
H(x, p)=\frac{p_{i} G_{i j} p_{j}}{\sqrt{p^{k} p_{k}}} .
$$

Applying (5), the steady HJ equation is derived. The steady HJ equation is solved by applying the wave front propagation algorithm using the Lax-Friedrichs scheme. In the other well-known approach, Parker et al. [3] and Melonakos et al. [5] derived the geodesic equation by minimizing,

$$
H(x, p)=\sqrt{\frac{\partial \varphi^{i}}{\partial x} G^{i j} \frac{\partial \varphi^{j}}{\partial x}},
$$

where $G^{i j}$ is the inverse of the Riemannian metric on the manifold. Recall that in our case it is the inverse of the diffusion tensor.

Notice that the wave front propagation approach solves the propagation of the fronts using (5), where the momenta are always perpendicular to them. Here, the wave equation is obtained from a local approach. A wave propagates according to Huygens' principle, where every point acts as a source. Thus, the fronts of the wave will evolve by locally propagating in every direction according to the diffusion tensor. The influence of the data in the entire domain will be limited. The optimal paths obtained from this approach can be interpreted as a generalization of the original streamline technique where the fiber evolves corresponding to a minimum of (4).

On the other hand, Hamiltonian (6) is obtained by means of a variational formulation that considers the entire domain and complete data to calculate the optimal curves. The resulting optimal paths will be those that globally minimize the cost, thus obtaining those minimizing the diffusion along the trajectory.

Both methods are robust to noise and can connect the initial point to any point inside the domain. Unfortunately, it is not clear which of both methods mentioned above suits better the real structures. Further research with validation on real structures should be done to check which of the methods is the most suitable in following fibers in the brain.

Recently, Sepasian et al. [8] introduced a new algorithm for geodesic fiber tracking using the EL approach. To find the geodesic connecting two given points on the boundary, the geodesic equation is derived in spherical coordinates, which reduces the number of ODEs to five, instead of the standard six first order ODEs. 
The solution of this new system of ODEs is solved by the two-point ray tracing method. This approach is properly dealing with the position when multi-valued arrival times occur. However, the work is represented only for unrealistic twodimensional synthetic data fields and one can show that the high-index derivation for three dimensional applications can lead to a Differential Algebraic Equations (DAE), which are computationally expensive to solve; see for example Brenan et al. [12].

\section{Governing Equations}

A geodesic connecting a pair of points on a Riemannian manifold is minimizing the length functional (4). Let $\underline{x}(\tau)=\left(x^{1}(\tau), x^{2}(\tau), x^{3}(\tau)\right)^{\top}$ be a point on a geodesic, where $\tau$ is a parameter along the geodesic. The solution to (4) satisfies the following system of three second order ODEs:

$$
\ddot{X}^{\gamma}+\Gamma_{\alpha \beta}^{\gamma} \dot{X}^{\alpha} \dot{X}^{\beta}=0, \quad \text { for } \quad \gamma=1,2,3,
$$

where $\Gamma_{\alpha \beta}^{\gamma}$ are the Christoffel symbols given by

$$
\Gamma_{\alpha \beta}^{\gamma}=\frac{1}{2}\left[g^{\gamma \sigma}\left(\frac{\partial}{\partial X^{\alpha}} g_{\beta \sigma}+\frac{\partial}{\partial X^{\beta}} g_{\alpha \sigma}-\frac{\partial}{X^{\sigma}} g_{\alpha \beta}\right)\right] .
$$

Here $g^{\gamma \sigma}$ represent the matrix component of $G^{-1}$ and $g_{\alpha \beta}$ the ones of $G$. In our application the metric $G$ is the inverse of diffusion tensor $D$. Let us define $u^{\gamma}(\tau):=\dot{X} \gamma(\tau)$ for $\gamma=1,2,3$, then we can rewrite equation (7) as follows,

$$
\begin{aligned}
\dot{X}^{\gamma} & =u^{\gamma}, \\
\dot{u}^{\gamma} & =-\Gamma_{\alpha \beta}^{\gamma} u^{\alpha} u^{\beta} .
\end{aligned}
$$

Consider the point $\left(x_{0}^{1}(\tau), x_{0}^{2}(\tau), x_{0}^{3}(\tau)\right)$ as the given initial point in the domain and $\left(u_{0}^{1}(\tau), u_{0}^{2}(\tau), u_{0}^{3}(\tau)\right)$ as an initial direction. We compute the solution to (9) for the given initial position and multiple initial directions using sophisticated ODE solvers, such as fourth order explicit Runge-Kutta. This gives us the set of geodesics connecting the given initial point to some points on the boundary. Depending on the application, two different post-processing approaches are possible. The first post-processing approach refers to applying the two-point ray tracing algorithm proposed in [8] in order to find the geodesic connecting two given points inside the domain. The second approach is to filter out the captured geodesics by applying connectivity measures. Here, we focus on the latter case.

The connectivity measure is used for finding a suitable trajectory corresponding best to real fiber bundles. We apply these measures in order to filter the geodesics, which are not holding strong connections between points on the domain. Since the fibers correspond to the geodesics connecting a pair of points in the Riemannian manifold, the most reasonable connectivity measure is the one that minimizes trajectories in a Riemannian manifold.

In recent papers, Astola et al. [9] and Parker et al. [13] represent the connectivity measures as ratio of lengths given by the Euclidean and Riemannian metric 
tensors. This measurement can be considered as a measure for the connection strength of a geodesic. The proposed measure reads,

$$
m_{L}(\underline{x})=\frac{\int_{0}^{\mathcal{T}}\left(\dot{X}^{\top} \dot{X}\right)^{1 / 2} d \tau}{\int_{0}^{\mathcal{T}}\left(\dot{X}^{\top} G \dot{X}\right)^{1 / 2} d \tau} .
$$

Note that locally, in anisotropic voxels this measure gives the maximum in the direction of the eigenvector corresponding to the largest eigenvalue; see Astola et al. [9].

\section{Numerical Model}

\subsection{Discretization}

The computational domain is discretized uniformly with grid size $h=1 \mathrm{~mm}$ and grid points $X_{i}=i h$ for $i=1,2,3, \ldots, N$ where $N$ is number of grid points in each direction. We label each grid point with a $3 \times 3$ tensor $G_{i j k}=D_{i j k}^{-1}$.

We approximate the metric derivatives at each grid point by using finite difference scheme such as the second order central scheme, i.e.,

$$
\frac{\partial g_{\beta \sigma}}{\partial x^{1}}\left(x_{i}^{1}, x_{j}^{2}, x_{k}^{3}\right) \approx \frac{1}{2 h}\left(g_{\beta \sigma}\left(x_{i}^{1}+h, x_{j}^{2}, x_{k}^{3}\right)-g_{\beta \sigma}\left(x_{i}^{1}-h, x_{j}^{2}, x_{k}^{3}\right)\right) .
$$

Second order one-sided differences are applied when the grid points are situated on the boundary, i.e,

$$
\begin{aligned}
& \frac{\partial g_{\beta \sigma}}{\partial x^{1}}\left(x_{0}^{1}, x_{j}^{2}, x_{k}^{3}\right) \approx \\
& \quad \frac{1}{2 h}\left(-3 g_{\beta \sigma}\left(x_{0}^{1}, x_{j}^{2}, x_{k}^{3}\right)+4 g_{\beta \sigma}\left(x_{1}^{1}, x_{j}^{2}, x_{k}^{3}\right)\right)-g_{\beta \sigma}\left(x_{2}^{1}, x_{j}^{2}, x_{k}^{3}\right), \\
& \frac{\partial g_{\beta \sigma}}{\partial x^{1}}\left(x_{0}^{1}, x_{j}^{2}, x_{k}^{3}\right) \approx \\
& \quad \frac{1}{2 h}\left(3 g_{\beta \sigma}\left(x_{N}^{1}, x_{j}^{2}, x_{k}^{3}\right)+4 g_{\beta \sigma}\left(x_{N-1}^{1}, x_{j}^{2}, x_{k}^{3}\right)\right)+g_{\beta \sigma}\left(x_{N-2}^{1}, x_{j}^{2}, x_{k}^{3}\right) .
\end{aligned}
$$

Note that similar expressions hold for derivatives with respect to $X^{2}$ and $X^{3}$. We apply trilinear interpolation for computing Christoffel symbols at any grid point on the domain.

\subsection{Results}

In this section we compute a geodesic for a discrete three-dimensional synthetic tensor field. Isotropic tensors are imposed as background excluding the influence of noise. In order to mimic real DTI acquisition, Rician noise is added to the $30 \times 50 \times 50$ synthetic image with $1 \times 1 \times 1 \mathrm{~mm}$ voxel size. For computing Rician noise, the signal attenuation is obtained for the noiseless diffusion tensors using the inverse of the Stejskal-Tanner relation, see [14]. The noise is 
a)

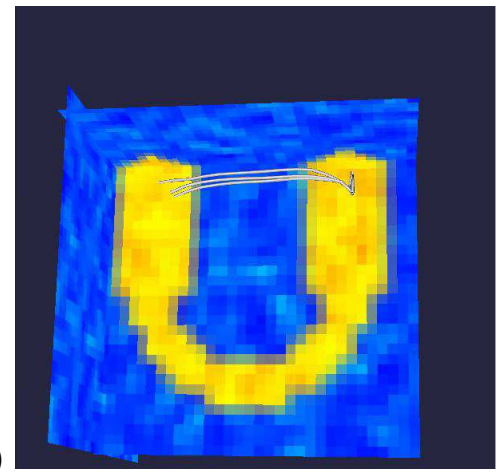

b)

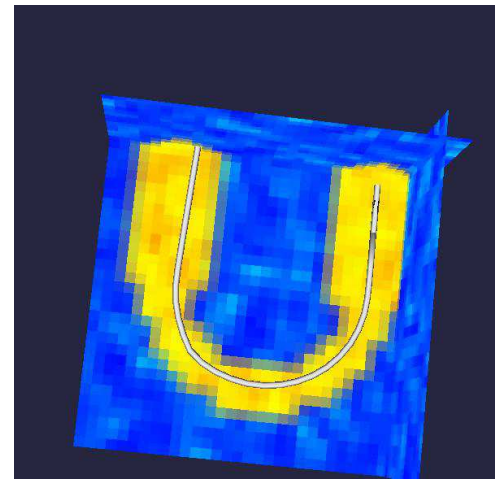

Fig. 2. Geodesic computed using HJ equation (a) and ray tracing algorithm (b).

added to each direction value with $S N R=15.3$ and the tensors are recomputed. Let us define the curved region as $R_{1}$ and the background as $R_{2}$, then tensors belonging to each region are computed with realistic eigenvalues, i.e., $\lambda_{R 1}=[3,17,17] \times 10^{-4} \mathrm{~mm}^{2} / \mathrm{s}$, and $\lambda_{R 2}=[7,7,7] \times 10^{-4} \mathrm{~mm}^{2} / \mathrm{s}$. Figure 2 shows the results corresponding to the U-shaped fiber using the HJ equation in $2 \mathrm{a}$ and ray tracing algorithm $2 \mathrm{~b}$. In order to find the best corresponding fibreous structure, the geodesic is computed by shooting rays from an initial point to all possible directions. Using connectivity measure (10) we select the strongest connection with the largest connectivity value.

\section{Conclusion}

In this paper, we have presented the geodesic based method for fiber tracking in diffusion tensor images. We reformulate the Euler-Lagrange form of the geodesic equation for three-dimensional metric-warped space and apply the ray tracing method to compute the solution. This new algorithm allows us to have more control over local orientation inside the domain. Moreover, there is no superposition principle for the Hamilton-Jacobi equation and the viscosity solution does not necessarily agree with the correct physical solution when multiple wave front arrivals occur in one position. While, in our approach by taking advantage of the EL form of geodesic equation that holds the superposition principle the multi-valued solutions can be captured. Results for realistic synthetic data has been shown for both HJ based fiber tracking and our proposed ray tracing algorithm. We show the potential of our method for capturing the correct tract corresponding to the underlying fiber structure. Assuming the reasonable cost for going through the correct fiber tract is a ratio between the Riemannian and the Euclidean length of the trajectory, we show that in the HJ approach the fiber tracking can collapse when the Euclidean and Riemannian lengths are proportional. 
We are interested in generalizing the algorithm for fiber tacking to higher resolution DTI, such as HARDI. Furthermore, we would like to extend the numerical scheme to overcome the diverging rays that may occur during the computations. Finally, since fibers can be computed independently, the algorithm is highly suitable for parallel computations.

\section{References}

1. Basser, P.J., Pierpaoli, C.: Microstructural and physiological features of tissues elucidated by quantitative-diffusion-tensor MRI. Journal of Magnetic Resonance 111(1) (1996) 209-219

2. Jun, Z., Hao, J., Ning, K., Ning, C.: Fiber tractography in diffusion tensor magnetic resonance imaging: A survey and beyond. Department of Computer Science, University of Kentucky, USA (2005)

3. Parker, G.J.M., Wheeler-Kingshott, C.A.M., Barker, G.J.: Distributed anatomical brain connectivity derived from diffusion tensor imaging. Information Processing in Medical Imaging 17th International Conference 2482 (2001) 106-120

4. Jackowski, M., Kao, C., Qui, M., Constable, R., Staib, L.: White matter tractography by anisotropic wavefront evolution and diffusion tensor imaging. Medical Image Analysis 9 (2005) 424-440

5. Melonakos, J., Mohan, V., Niethammer, M., Smith, K., Kubicki, M., Tannenbaum, A.: Finsler tracktography for white matter connectivity analysis of the singulum bundle. MICCAI, Springer (2007)

6. Parker, G., Luzzi, S., Alexander, D., Wheeler-Kingshott, C., Ciccarelli, O., Lambon-Ralph, M.: Lateralization of ventral and dorsal auditory-language pathways in the human brain. NeuroImage 24 (2007) 656-666

7. Jbabdi, S., Bellec, P., Toro, R., Daunizeau, J., Pélégrini-Issac, M., Benali, H.: Accurate anisotropic fast marching for diffusion-based geodesic tractography. International Journal of Biomedical Imaging (2008)

8. Sepasian, N., Vilanova, A., Florack, L., ter Haar Romeny, B.: A ray tracing method for geodesic based tractography in diffusion tensor. Workshop on Mathematical Methods in Biomedical Image Analysis (MMBIA) (2008)

9. Astola, L.J., Florack, L.M.J., ter Haar Romeny, B.M.: Measures for pathway analysis in brain white matter using diffusion tensor imaging. In Proceeding of Information Processing in Medical Imaging, Lecture Notes in Computer Science (2007)

10. C. Lenglet, R. Deriche, O.F.: Inferring white matter geometry from diffusion tensor mri: Application to connectivity mapping. Proc. 8th European Conference on Computer Vision (2004) 127-140

11. Runborg, O.: Mathematical models and numerical methods for high frequency waves. Communications in Computational Physics 2 (2007) 827-880

12. Brenan, K.E., Campbell, S.L.V., Petzold, L.R.: Numerical solution of initial-value problems in differential-algebraic equations. 2 (1996)

13. Prados, E., Soatto, S., Lenglet, C., Pons, J.P., Wotawa, N., Deriche, R., Faugeras, O., Soatto, S.: Control theory and fast marching techniques for brain connectivity mapping. IEEE computer society press 1(1) (2006) 1076-1083

14. Stejskal, E.O., Tanner, J.E.: Spin diffusion measurements: Spin echoes in the presence of a time-dependent field gradient. The Journal of Chemical Physics 42(1) (1965) 288-292 